\title{
Legislación con enfoque de Derechos Humanos para la gestión de Desarrollo Humano Sostenible en Honduras
}

\author{
Abog. Javier David López Padillla*
}

\section{Resumen}

El presente trabajo tiene como propósito principal aportar, desde la Ciencia Jurídica, argumentos para desarrollar una propuesta que contribuya a mejorar las oportunidades del Desarrollo Humano Sostenible (en adelante DHS) en Honduras. Esta contribución académica nace del reconocimiento de la función de la Ley, como categoría jurídica y social ordenadora de las relaciones humanas en un contexto histórico determinado. Dicho reconocimiento sobre la función social de la Ley se refiere al marco de atribuciones y restricciones al que el estado de Honduras (en su carácter de organización política) debe ajustar sus decisiones, especialmente en la planificación del DHS.

El objetivo principal de esta investigación es fortalecer al sistema jurídico nacional, reconociendo la relación entre las normas legales y el DHS en su condición de derecho humano, enfatizando la necesidad de proveer criterios para la generación de comunicación de las demandas sociales en el proceso de creación normativa por medio del enfoque de derechos humanos.

El proceso de investigación desarrollado en el presente documento toma el enfoque del tridimensionalismo jurídico, en el cual se destacan elementos propios de las investigaciones socio-jurídicas cualitativas, de análisis crítico social del discurso legal constitucional, con inclusión de aplicaciones propias del Derecho Comparado.

Uno de los principales hallazgos de este trabajo, alude a que la mayor cantidad de leyes en Honduras están enfocadas en los ámbitos administrativo-fiscal, mercantil y civil; y en cuarto lugar lo penal. Es necesario considerar que estos cuerpos normativos regulan en esencia la dimensión económica de la sociedad; para el caso, desde el punto de vista fiscal, se establecen los criterios que orientan al Estado en su función de recaudación de impuestos, la que a su vez está relacionada con la capacidad adquisitiva de los contribuyentes. La imposición de tributos es una prerrogativa de los Estados basada en la contribución que todos los ciudadanos de un país están llamados a prestar para mejorar las condiciones de vida social de la comunidad a la que pertenecen. En el caso de las esferas mercantil y civil, sus contenidos normativos regulan las actividades de las personas en su ámbito privado, y en especial, en la relación con el ejercicio del Derecho de Propiedad. Esto quiere decir que históricamente, la preocupación del legislador ha estado concentrada en la actividad económica de las personas que regula mediante las normas que promulga, ya sea en razón del ejercicio del derecho de propiedad para el uso, goce y disposición privada de los bienes sobre los que ejerce este derecho, o para la regulación de la explotación masiva del mismo y/o para la imposición de tributos en razón del patrimonio de las personas.

Palabras Clave: Desarrollo, Derecho, Derechos Humanos, Ley.

Fecha de Recepción: viernes 03 de junio 2016

Fecha de Aprobación: viernes 25 de noviembre 2016

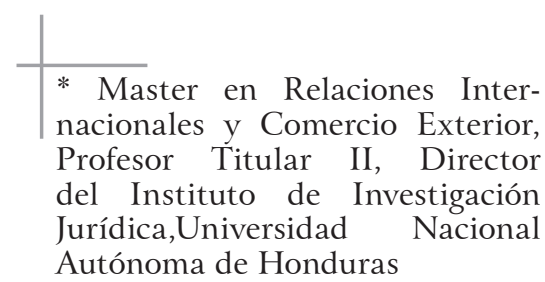

Autónoma de Honduras 


\title{
Legislation with a Human Rights approach for the Management of Sustainable Human Development in Honduras.
}

\author{
Abog. Javier David López Padillla*
}

\begin{abstract}
The study of the principle of most general shareholder together allows us to perform a detailed analysis This work has as main purpose providing arguments from legal science to develop a proposal that will help to improve the opportunities of Sustainable Human Development in Honduras DHS, (Desarrollo Humano Sostenible). This academic contribution comes from the recognition of the role of law as a legal category and social ordering of human relations in a particular historical context. Such recognition about the social function of the Law refers (and extends) the framework of powers and restrictions to which the State of Honduras (in its capacity as political organization) must adjust their decisions specially refer to the DHS planning.

The main objective of this research is to strengthen the national legal system recognizing the relationship between legal standards and DHS in its capacity as Human Right, emphasizing the need to provide criteria for the generation of communication of social demands in the process of creating legislation through the approach of Human Rights.
\end{abstract}

The research process developed in this paper is positioned in the approach of the legal tridimensionalism, which highlights elements of the socio-legal qualitative research critical social analysis of legal discourse constitutional, including own applications of comparative law. One of the main findings of this study refers to as many laws in Honduras are concentrated in fiscal - administrative, commercial civil; and fourthly criminal. It is necessary to consider that these regulatory bodies regulate essentially the economic dimension of society; in case the criteria that guide the state in its tax collection function are established, from the fiscal point of view, which in turn is related to the purchasing power of taxpayers.

The imposition of taxes is the prerogative of States based on the contribution that all citizens of a country are called to render to improve the social life of the community to which they belong. In the case of commercial and civil spheres, their normative content regulate the activities of individuals in their private sphere, especially in connection with the exercise of the property right. This means that historically, the legislature's concern has been focused on the economic activity of persons regulated by the rules enacted, either because of the exercise of the right of property for the use, enjoyment and private disposition of property on those who exercise this law, or regulation of the massive exploitation of the same and / or the imposition of taxes due to the heritage of the people.

Key Words:Development, Law, Human Rights.

* Master en Relaciones Internacionales y Comercio Exterior, Profesor Titular II, Director del Instituto de Investigación Jurídica,Universidad Nacional Autónoma de Honduras

Fecha de Recepción: viernes 03 de junio 2016

Fecha de Aprobación: viernes 25 de noviembre 2016 


\section{Introducción}

El presente trabajo de investigación tiene como objetivo eLa innto de partida el reconocimiento de la relación entre el derecho y el desarrollo, cuya teorización viene desarrollándose desde mediados del siglo $\mathrm{XX}, \mathrm{y}$ que atribuye a la ley, como fuente del derecho, la condición de ser la línea de orientación y el criterio de legitimación de las acciones y decisiones que un Estado asume a la hora de gestionar las acciones conducentes al DHS.

Normalmente (junto con otras consideraciones) a la gestión de un Estado basada en la Ley se le conoce como el Estado de Derecho, por tanto ésta investigación fundamenta teóricamente sus argumentaciones en las nociones generales antes referidas y pondera críticamente el contexto normativo actual en relación con los conceptos generales de ley, vigencia de la ley, Estado de Derecho, Derechos Humanos, Gobernabilidad y DHS.

El estudio de la relación entre derecho y desarrollo ha sido dinámico y evolutivo en razón de la naturaleza compleja de ambas categorías que no pueden determinarse definitivamente sino que se nutren de los contextos sociales cambiantes donde se despliegan. La Ley como categoría principal del derecho posee en su naturaleza esta impronta dinámica y evolutiva compuesta al menos por tres dimensiones, a saber: las normas legales, promulgadas por un Estado; los hechos sociales que dichas normas ordenan y los valores que las mismas persiguen realizar.

Históricamente en Honduras, la relación entre derecho y desarrollo nunca ha sido muy clara, su fundamentación empírica se da sobre la noción de la función de la Ley como expresión o fuente del derecho y como medio de regulación de las fuerzas sociales, económicas y políticas presentes en un contexto social determinado. Dicha relación está, a su vez, condicionada a la complejidad de los contenidos que la componen, lo que conlleva la dificultad en obtener un concepto unívoco o estático en la construcción de una línea depensamiento que integre las aspiraciones civiles, políticas, económicas, sociales y culturales del país.

El derecho y las leyes no constituyen en sí mismas opciones al DHS. Los estadios deseados de desarrollo son alcanzados por la interacción de la acción política que define los modelos pertinentes contextualmente, así como la instrumentación económica -el uso de los recursos-, para hacer factibles o viables los modelos escogidos y que se apoya en el derecho, particularmente en las leyes, para normativizar y dar un sentido de certeza, equidad y predictibilidad a la acción de los tomadores de decisiones en los procesos de uso y distribución, con sentido de justicia, de los recursos.

Las instituciones internacionales con mandato para cono- cer sobre el tema de desarrollo, así como las universidades y demás espacios de pensamiento científico (político, sociológico y económico), coinciden que los retos que enfrentan los países en vías de desarrollo son, en muchos aspectos, comunes entre ellos. Existe un dato histórico que informa sobre las distintas etapas y características en que se fueron presentando, a nivel mundial, las tendencias del desarrollo, desde las que implicaban un esfuerzo eminentemente economicista, hasta las que fueron incorporando las categorías de sustentabilidad y humanización que hoy por hoy se conocen.

El consenso político y social para erradicar progresivamente la pobreza y la vulnerabilidad ha sido la motivación de todas las iniciativas estatales para identificar caminos conducentes al DHS. En un momento determinado de su historia, los países han decidido implementar medidas económicas y políticas para mejorar las condiciones de vida de sus habitantes, medidas que al inicio se identifican con la nivelación de estándares de educación, salud, vivienda, acceso al agua potable, etc.

En este punto, diversos autores coinciden (Seidmann, Seidmann, \& Abeysekerer, 2001) en que unos países han sido más exitosos que otros en lograr los objetivos de desarrollo trazados, especialmente en el transcurso del siglo XX. Así, encontramos, por ejemplo, países que a pesar de haber sido afectados por los eventos de las dos guerras mundiales, hoy cuentan con estándares de vida comparativamente más altos que los de otros países, que si bien no han sido víctimas directas de los eventos de guerra mencionados, han sufrido procesos internos de acomodación política y social que hasta el día de hoy no han logrado superar. Sin embargo, la historia también documenta que los retos que viven actualmente estos países en vías de Desarrollo han sido superados antes por otros más avanzados en el camino y de los cuales pueden tomarse selectivamente algunas lecciones aprendidas.

Una de estas lecciones aprendidas por los países que gozan actualmente de mejores condiciones de desarrollo tiene que ver con la necesaria fundamentación de sus acciones y decisiones políticas en normas legales creadas por sus legisladores, que no sólo han contado con una fortaleza técnica en su creación, sino que han sido formuladas mediante procesos participativos y transparentes.

A finales del siglo $X X$, la categoría Desarrollo adquirió otro significado al haber sido asumida como un Derecho Humano por la comunidad internacional, en el marco de la Organización de las Naciones Unidas, a saber (ONU, 1986):

"un derecho humano inalienable, en virtud del cual todo ser humano y todos los pueblos están facultados para participar en un desarrollo económico, social, cultural y político en el que puedan realizar plenamente todos los derechos humanos y libertades fundamentales; a con- 
tribuir a ese desarrollo y a disfrutar de él... implica también la plena realización de los pueblos a la libre determinación, que incluye, con sujeción a las disposiciones pertinentes de ambos Pactos internacionales de derechos humanos, el ejercicio de su derecho inalienable a la plena soberanía sobre todas sus riquezas y recursos naturales".

En ese sentido, se entiende que el concepto de Desarrollo pasó de ser una categoría primordialmente económica para convertirse en una con relevancia jurídica puesto que su consideración de "Derecho", supone la existencia de una relación entre sujetos de derechos y sujetos de obligaciones, expresada en la ley. Es así que con la presente investigación se analiza el rol de la Ley como agente estratégico para el goce del Derecho al Desarrollo en el concepto asumido por la comunidad internacional. En su contenido se describe el problema a investigar, su justificación, los objetivos generales y específicos, las referencias teóricas que guían el entendimiento del tema y sirven para interpretar los resultados del trabajo, la propuesta metodológica correspondiente y finalmente los resultados del proceso de análisis que tiene como producto final la proposición de un modelo para mejorar los procesos legislativos que coadyuven a la gestión del Desarrollo Humano Sostenible.

\section{Metodología}

El proceso de investigación desarrollado en el presente documento se posiciona en el enfoque del tridimensionalismo jurídico, en el cual se destacan elementos propios de las investigaciones socio-jurídicas cualitativas, de análisis crítico social del discurso legal constitucional, con inclusión de aplicaciones propias del derecho comparado.

El posicionamiento "tridimensional" del derecho permite entender e interpretar la realidad jurídica desde tres perspectivas inter- vinculadas de la experiencia social y legal, a saber, la dimensión de las normas promulgadas

por el estado de Honduras que ordenan la vida social; la dimensión de los valores que se pretenden realizar por medio de las normas legales, y que en la presente investigación se subsumen en las categorías de los derechos humanos internacionalmente reconocidos, y finalmente, la dimensión fáctica, que constituye la fuente de datos de la realidad social que se pretende regular mediante las normas en atención a los valores. Una de las ventajas del enfoque metodológico del tridimensionalismo jurídico es que compatibiliza la posibilidad de desarrollar investigaciones y propuestas teóricas individuales desde los campos de la sociología, el derecho y la filosofía, variando su enfoque en el acento que cada disciplina le imprima. En otro sentido, también permite la generación teórica de abordajes multi-disciplinarios porque reconoce la complemen- tariedad compleja de las tres dimensiones (normas, hechos y valores). En ese sentido, las premisas sobre las que descansa esta postura pueden expresarse de la siguiente manera:

a. Las normas legales son categorías que generan o se derivan de hechos sociales.

b. Las normas legales son categorías que generan 0 derivan valores humanos.

c. Los hechos sociales son categorías que generan o derivan normas legales.

d. Los hechos sociales son categorías que generan 0 derivan valores humanos.

e. Los valores humanos son categorías que generan 0 derivan normas legales; $y$,

f. Los valores humanos son categorías que generan 0 derivan hechos sociales.

De manera particular, en el caso de la presente investigación se toma como punto de partida el reconocimiento de la relación entre las categorías de derecho y desarrollo, la invocación del enfoque del tridimensionalismo jurídico resulta pertinente, especialmente cuando la atención de la tesis recae sobre el fenómeno del desarrollo, ya que permite entenderlo desde las mismas dimensiones del enfoque tridimensional, es decir, el desarrollo como derecho, como hecho social y como valor. En un intento de representar gráficamente lo dicho en las líneas anteriores, se presenta el siguiente esquema que condensa la postura metodológica del presente estudio:

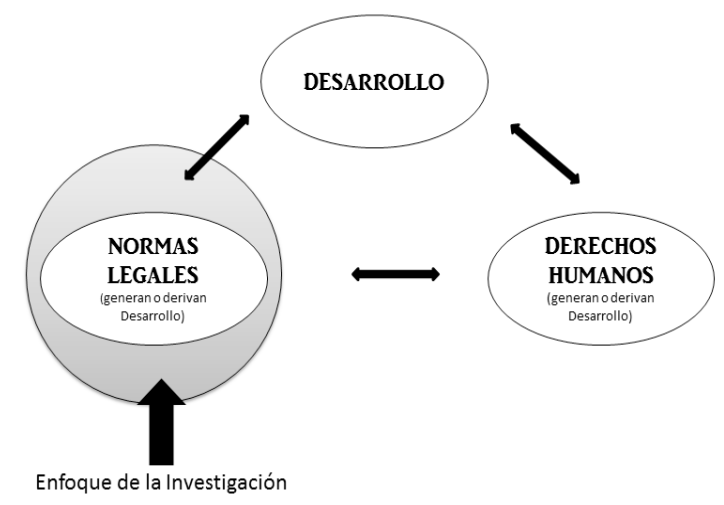

Figura 1. Elaboración Propia. Representación gráfica de la relación del tridimensionalismo jurídico y el desarrollo.

\section{Sobre EI Desarrollo Humano Sostenible}

La noción del desarrollo es el resultado de un proceso de definición, progresivo e inacabado, que partió de un enfoque estrictamente economicista (por ejemplo, las teorías de crecimiento económico, entre otras) hacia otros que se preocupan 


\section{Legislación con enfoque de Derechos Humanos para la}

gestión de Desarrollo Humano Sostenible en Honduras

por visibilizar la centralidad del ser humano como "agente" y beneficiario de las acciones para el desarrollo (la propuesta del capital social, desarrollo humano, etc.), hasta llegar a aquellos que rescatan la necesidad de incluir en el dialogo la dimensión ecológica- sostenible (el enfoque del Desarrollo Humano Sostenible). El largo camino recorrido por los estudiosos de estos temas, hasta llegar a una definición integral, no ha terminado todavía debido a las múltiples y complejas variables que se adhieren en la medida que se refuerza, desde distintas dimensiones (históricas, políticas, culturales, económicas, sociales, religiosas, etc.), la posición del ser humano.

El interés por el Desarrollo Humano no es nuevo, tal como se establece en el Primer Informe sobre Desarrollo Humano del Programa de las Naciones Unidas para el Desarrollo (Programa de las Naciones Unidas para el Desarrollo, PNUD), 1990), dicho interés ha sido la preocupación motivadora presente en los escritos de los fundadores de la economía cuantitativa (William Petty, Grerory King, Francois Quesnay, Antoine Lavoisier y Joseph Lagrange) así como de la economía política (Adam Smith, David Ricardo, Robert Malthus, Karl Marx y John Stuart Mill), (Anand \& Sen, Human Development Reports, 1994).

A Sudhir Anand y Amartya Sen (Anand \& Sen, Human Development Reports, 1994) se les reconoce internacionalmente como los primeros proponentes académicos del concepto de Desarrollo-Humano-Sostenible a partir de la publicación conjunta que presentaron en 1994 en la Universidad de Harvard, conocida como "Sustainable Human Development: Concepts and Priorities". En la parte introductoria de dicha publicación se proponen los contenidos para cada una de las tres categorías del concepto, a saber: 1) El Desarrollo; 2) Lo humano; y 3) Lo sostenible. En cuanto a la categoría de Desarrollo, toman como punto de partida la coincidencia que reconocen en los escritos de 1792 de Mary Wollstonecraft (en "A vindication of the rights of woman") y Thomas Paine (en "The rights of man") en los cuales ambos autores la definen como "el poder de las personas sobre sus propias vidas y oportunidades para vivir de la manera en la cual tienen razones para valorarlas" 1

Sobre la dimensión de lo humano, los autores citados elaboran sus ideas a partir de la universalidad del desarrollo, en la cual el reconocimiento de la potestad humana para vivir de la manera que considera valiosa, debe realizarse para todos, sin condiciones de clase social, género, raza o edad, etc. Finalmente, sobre la dimensión de lo sostenible ${ }^{2}$, los autores

\footnotetext{
1- De la traducción literal en inglés de "the power over their own lives and opportunities to live the way they had reasons to value ", en (Anand \& Sen, Human Development Reports, 1994).
}

2-El uso de la frase "Desarrollo Sostenible" se encuentra desarrollado por primera vez en el documento "Nuestro Futuro Común" también conocido como "Informe Brundtland" de la Comisión Mundial sobre Medio Ambiente y Desarrollo de las Naciones Unidas. En él se plantea la posibilidad de obtener un crecimiento económico basado en políticas de sostenibilidad y expansión de la base de recursos ambientales. Este Informe definió al proponen proponen la consideración sobre la garantía de los int las futuras generaciones, en la misma medida en la que se intenta asegurar los intereses de las presentes generaciones; la visión prospectiva del futuro demanda el respeto, en la misma medida, de las oportunidades para la generación del presente; igualmente establecen que no es una obligación mantener el mundo tal cual la generación presente lo encontró, si no, conservar las oportunidades para que las generaciones futuras vivan una vida valiosa.

Nótese que en el caso de las dimensiones de lo humano y lo sostenible se descubre una vinculación interesante ya que el aseguramiento de los intereses de las generaciones futuras es una forma de universalizar el Desarrollo, y, a la vez, una demanda de la aplicación imparcial del mismo, dentro y entre generaciones.

Los autores anteriormente citados también establecen una importante diferenciación sobre las dos posiciones desde las que la economía ha intentado estudiar el Desarrollo Humano (Anand \& Sen, Human Development Reports, 1994); en un primer momento reconocen la perspectiva de análisis económico del desarrollo centrada en la maximización de la riqueza; y en otro momento reconocen la perspectiva de análisis enfocada en el mejoramiento de la calidad de vida de la personas.

Una de las conclusiones más importantes a las que llegan los autores a partir de la diferenciación de esas dos tendencias económicas, es la identificación de una relación de colaboración mutua entre ambas, lo que no ignora sus luchas en la búsqueda de una posición académica/política hegemónica. Sin embargo, dejan entrever que por sí solas no han sido capaces de consolidar el Desarrollo Humano. En su criterio, numerosos estudios comparativos demuestran que en países como Costa Rica, Jamaica, China, entre otros, las dificultades en el crecimiento del Producto Interno Bruto (PIB), por ejemplo, no han incidido ni directa, ni negativamente en índices tales como la esperanza de vida, mortalidad infantil, alfabetismo, etc.; lo que no debe interpretarse como una negación de la instrumentalidad de la maximización de la riqueza, ya que también existe evidencia comparativa que confirma en el largo plazo la relación entre mejores condiciones de vida y crecimiento económico.

El enfoque en el mejoramiento de la calidad de vida de las personas, le otorga especial importancia a la implicación de la dimensión pública en la promoción del desarrollo humano así como en la distribución de los ingresos adicionales para los pobres; considera el carácter contingente de la riqueza

Desarrollo como la satisfacción de las necesidades del presente sin comprometer las oportunidades de las generaciones futuras en el logro de sus propias necesidades. 
como instrumento de desarrollo y niega que ésta sea el único medio para realizarlo.

Los autores también destacan un elemento adicional al concepto de Desarrollo Humano Sostenible referido a lo institucional (Anand \& Sen, Human Development Reports, 1994), en ese sentido, argumentan que la calidad de vida que una persona puede disfrutar no depende únicamente del ejercicio de su iniciativa individual sino también de las circunstancias sociales dependientes de condiciones institucionales. Por tanto, se descubre una relación de complementariedad entre la agencia individual y el apoyo institucional crucial para el logro de las oportunidades individuales en diferentes campos, incluyendo la capacidad de conducir satisfactoriamente una vida digna de vivir. Asimismo, se identifica que la realización de la dimensión de sostenibilidad está apoyada por condiciones institucionales sistemáticas, es decir que se requiere de la intervención institucional para permitir que el agenciamiento individual tome nota de los intereses y derechos de las generaciones futuras.

La tesis de los autores se resume al establecer que el Desarrollo Humano Sostenible tiene significado doble, como medio y como fin; en el primer caso considera la contribución de las personas en la consecución del bien común y la prosperidad; en el segundo caso, privilegia la realización individual en la construcción de una vida digna de vivir.

En el año 2000, Sudhir Anand y Amartya Sen en otro artículo denominado Human Development and Economic Sustainability (Anand \& Sen, 2000) hicieron una revisión de su primer escrito Sustainable Human Development: Concepts and Priorities (Anand \& Sen, Human Development Reports, 1994) referido en párrafos anteriores, en el que refuerzan la idea que el crecimiento económico no es la única vía ni la más idónea para permitir el desarrollo humano, concretizan que esta dimensión tiene un carácter contingente al desarrollo en relación al uso que se haga de la riqueza generada y que su condición de "medio" para el desarrollo es compartida con otras dimensiones tales como la organización social, entre otras, sin perder de vista la consideración de escenarios para garantizar la sostenibilidad de dicho desarrollo entre generaciones presentes y futuras y entendiendo dicha "sostenibilidad" como la capacidad de mantener el "agenciamiento" humano.

Cabe rescatar que el concepto de Desarrollo Humano Sostenible encierra en su esencia otras dimensiones pocos estudiadas, entre las cuales destaca el papel de la coordinación institucional. El enfoque parte de la idea que el aseguramiento de las oportunidades -intra- e inter-generacionales no es un acto de caridad sino de justicia, que se realiza de una forma distributiva, no discriminatoria, mediante la activación de la organización social, elementos todos que corresponden al interés del Derecho.
Jorge Witker Velásquez y Rogelio Larios Velasco, en su trabajo "Metodología Jurídica" (Witker y Larios, 2002) citando a su vez la obra

Introducción al estudio del Derecho del autor Eduardo García Maynez, proponen un concepto de Derecho en los siguientes términos:

\begin{abstract}
"El Derecho es un hecho cultural, en cuanto expresa la preocupación del individuo social por mantener el precepto que garantiza la convivencia y da origen a instituciones nuevas pero necesarias para el bien común... El Derecho como ciencia social está sujeto a un proceso que se inicia con el nacimiento de las instituciones que regulan la conducta humana, que se perfeccionan mediante el análisis de la historia y la reflexión, y se extinguen o desaparecen, cuando se tornan inútiles o se juzgan manifiestamente injustas".
\end{abstract}

De dicho concepto puede destacarse la existencia de tres categorías que conforman al Derecho: los hechos, los valores y las normas. Dicho de otra manera, al seccionar el concepto citado anteriormente se identifican tres categorías que constituyen al derecho y lo convierten en una realidad trivalente 0 tridimensional (3D), dichas categorías corresponden a lo fáctico, estimativo y normativo.

Figura 2. Elaboración Propia. Identificación de las tres categorías propuestas por el tridimensionalismo jurídico.

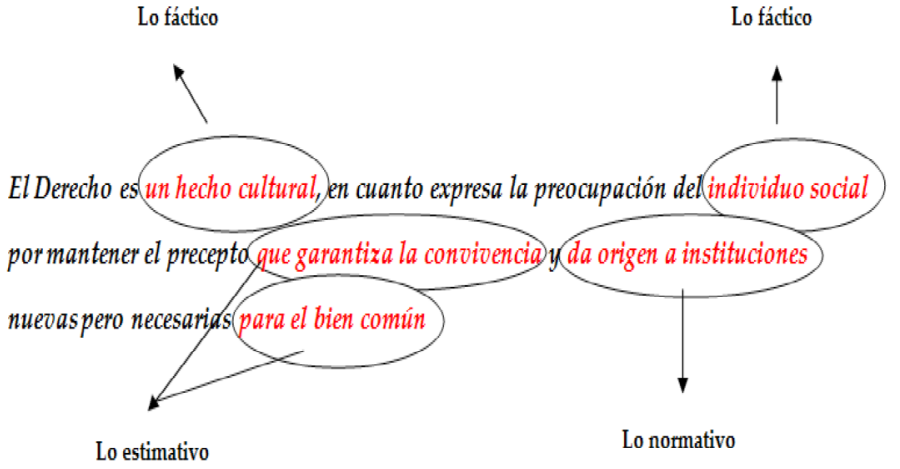

Siguiendo a Bartolo Cepeda (Cepeda, 1999) esta forma de pensamiento promueve una concepción integral del Derecho en la que deben valorarse inseparablemente las dimensiones que lo componen, a saber, la norma, el hecho y el valor; mismas que corresponden respectivamente a las categorías de eficacia -realizada en las normas-, validez extrínseca -realizada en los hechos-, y validez

intrínseca -realizada en los valores-, que constituyen el fenómeno jurídico.

Bartolo Cepeda (Cepeda, 1999), continua afirmando que esta concepción tridimensional del derecho tiene su fundamento en la naturaleza humana ya que hace una representación 
sintética del ser y el deber ser. Bajo esta idea, el tridimensionalismo jurídico presenta al derecho desde una perspectiva realista y total, a partir del mundo jurídico de las normas, de las conductas sociales y de los valores. Con ello, esta forma de asumir el derecho intenta sobreponerse a explicaciones reduccionistas del fenómeno jurídico, evitando el error de considerarlo únicamente como un mero fenómeno normativo, sociológico o axiológico, y por tanto debiendo considerarse como un hecho social con forma normativa y con referencia a un cierto orden de valores.

A Miguel Reale se le reconoce la acuñación del término "tridimensionalismo jurídico" (Reale, 1989). Bajo su perspectiva, se considera que el derecho representa una síntesis del ser y del deber ser, y que su fundamento está en la naturaleza humana. Bartolo Cepeda (Cepeda, 1999), siguiendo a Reale manifiesta que esta postura "propone enfocar el derecho con una perspectiva realista y total, a partir de la observación del mundo jurídico de las normas, de las conductas sociales y de los valores:

\begin{abstract}
...el Derecho es una realidad, digámoslo así, trivalente, o, en otras palabras, tridimensional. es simple hecho, valor y norma, para quien quiera que lo estudie, dándose tan solo una variación en el ángulo o prisma de investigación. La diferencia es, pues, de orden metodológico, según el objetivo que se tenga en mente alcanzar. Es lo que con agudeza Aristóteles llamaba 'diferencia específica', de tal modo que el discurso del jurista va del hecho al valor y culmina en la norma; el discurso del sociólogo, va de la norma hacia el valor, y culmina en el hecho, y finalmente, nosotros ${ }^{3}$ podemos ir del hecho a la norma, culminado en el valor, que es siempre una modalidad del valor de lo justo, objeto propio de la filosofía del Derecho..."
\end{abstract}

En el mismo sentido, establece que la teoría tridimensional del derecho permite una representación simbólica de los datos que componen la experiencia jurídica, los correlaciona dialécticamente de manera que los tres factores competentes se desarrollen interrelacionados en el tiempo, con la posibilidad de preverse, aunque sea de forma conjetural. Afirma que podrán influirse de manera recíproca incluso en función de la supervivencia de nuevas mutaciones factuales, axiológicas y normativas.

Según Miguel Reale (Reale, 1989) una regla de derecho positivo puede considerarse perfecta cuando la promulga una autoridad legítima con base en el bien común, y encuentra, en virtud de su valor ético y de su racionalidad, la adhesión 0 asentimiento de los miembros de una comunidad. En esta regla se integran, plenamente armonizadas, la validez ética, la sociológica y la formal o técnico jurídica.

3- Al decir "nosotros" se refiere a sus colegas de filosofía del Derecho de la Universidad de Sao Pablo, Brasil.
Con base a lo descrito anteriormente puede concluirse que todo fenómeno jurídico implica necesariamente un hecho subyacente (de naturaleza económica, social, técnica, etc.), un valor que confiere determinada significación a ese hecho que inclina la acción de las personas a lograr cierto propósito y la existencia de una norma que representa la relación o medida que vincula los hechos y las aspiraciones. Dichas dimensiones no existen separadamente unas de otras, sino que coexisten en una unidad concreta donde se exigen recíprocamente y actúan como "los elementos de un proceso (el derecho es una realidad histórico-cultural) de tal modo que la vida del derecho resulta de la interacción dinámica y dialéctica de los tres elementos que la integran" (Reale, 1989).

\section{Sobre la relacion entre el Derecho y el Desarrollo}

Carlos Rodríguez (Rodríguez Braun, 2005) y German Burgos (Burgos, 2009) coinciden en la noción que el estudio de la relación entre el Derecho y el Desarrollo no es reciente,

\begin{abstract}
"Adam Smith había sostenido que el funcionamiento del mercado en cuanto generador de bienestar colectivo, suponía no sólo la acción organizador de la mano invisible, sino la existencia de un aparato de justicia resolutor de los conflictos y a cargo del Estado... Por su parte para la economía neocláisca, si bien el mercado tendía al equilibrio, se daba por sentado que para ello era necesario el funcionamiento adecuado de las instituciones públicas encargadas del orden, la garantía de los derechos de propiedad y el cumplimiento de los contratos".
\end{abstract}

De acuerdo con David Trubek y Alvaro Santos (Trubek y Santos, 2008), el estudio de la relación entre el Derecho y el Desarrollo se registra a partir del S. XIX principalmente en algunos estudios de Marx y Weber ${ }^{4}$. Sin embargo, es hasta después de la Segunda Guerra Mundial en Estados Unidos, que se comienzan a desarrollar reflexiones más organizadas y sistemáticas en este campo, las que al inicio implicaban la propuesta de reforma de los sistemas legales como parte de la actividad de las agencias internacionales de desarrollo que consideraban al Derecho como un instrumento de los Estados para generar crecimiento económico.

En la década de 1980, el interés de estudio sobre la relación

4- David Trubek (Trubek, 2014) argumenta que en los estudios de Sociología del Derecho de Max Weber se identifica la idea que el sistema legal europeo era un factor importante para el desarrollo del capitalismo y a partir de esa premisa se comienza a construir la teoría sobre el Derecho y el Desarrollo.

Por su parte German Burgos (Burgos, 2009) establece que el "interés actual por la relevancia del Derecho en el Desarrollo cuenta con un antecedente fundamental expresado en los estudios realizados por Max Weber .. siendo así que uno de los padres de la sociología moderna abordó, a finales del s. XIX, el estudio de la relación entre Derecho y Economía y, en particular trató de identificar el papel que cumplió el derecho racional moderno en la implantación y desarrollo exitoso de la economía capitalista". 
entre derecho y desarrollo creció, enfatizándose el rol de la ley como marco para el desarrollo de actividades de mercado y en la década de 1990 se generó una nueva aproximación que posicionaba a la ley como el instrumento para corregir las fallas del mercado y garante del crecimiento económico. Después de esta época comienza a utilizarse el término "Estado de Derecho" no sólo como una herramienta para la gestión del Desarrollo, sino como un objetivo en sí mismo para asegurar el desarrollo.

La teoría del derecho y desarrollo identifica tres etapas de su proceso de formación, a saber:

1. 1965-1980: Conocida como el "Estado de Derecho y Desarrollo" se caracteriza por dos ideas: 1) que las políticas de desarrollo estaban enfocadas en el rol del Estado como "gerente" de la economía y, 2) la preocupación por la transformación de las sociedades tradicionales.

Los agentes de las instituciones internacionales de desarrollo asumieron que la Ley podía ser utilizada como una herramienta para el crecimiento económico. Al inicio estas consideraciones no fueron muy evidentes pero posteriormente emergieron como propuesta doctrinaria que promovía la intervención de los Estados en la economía mediante la creación de instrumentos legales que viabilizaran dicha intervención.

La ley se consideró como una herramienta para eliminar las barreras tradicionales y lograr el cambio del comportamiento económico, canalizando el control macro-económico del Estado de acuerdo a una planificación nacional. Asimismo, en esta etapa se consideró que la ley era necesaria para crear el marco de operación de una burocracia eficiente y asegurar la gobernabilidad de las empresas del sector público, lo que implicó a su vez, la actualización del Derecho Público y la replicación de modelos regulatorios establecidos en los países desarrollados.

2. 1980-2000: Conocida como la etapa del "Derecho y el Mercado Neoliberal", se caracteriza por la consideración que la mejor forma de lograr el crecimiento era mediante la obtención del "precio correcto" en las relaciones de mercado, promoviendo la disciplina fiscal y removiendo las distorsiones creadas por la intervención del Estado en la economía, asegurando el libre comercio y la inversión extranjera.

La percepción de la ley en esta etapa era la de constituir un instrumento para asegurar las transacciones privadas, poniendo énfasis ya no en el derecho público sino en el privado a fin de proteger la propiedad privada y facilitar los intercambios contractuales. La ley fue utilizada para establecer límites estrictos de intervención estatal en la economía y asegurar un tratamiento igualitario a la inversión extranjera.
Las reformas legales impulsadas en esta etapa tenían que ver el fortalecimiento de los derechos de propiedad y los derivados de las relaciones de contratos comerciales, así como la definición de roles de los poderes judiciales que garantizaran los límites de la intervención estatal en las actividades comerciales privadas y la facilitación de la actividad en el mercado. A partir de esta etapa se implanta la idea que la independencia de los tribunales de justicia provee fidelidad y predictibilidad a las decisiones que impactan las relaciones de mercado.

3. 2000-a la fecha: Las escuelas de pensamiento sobre Derecho y Desarrollo consideran que nos encontramos en el proceso de una tercera etapa que constituye a la vez un nuevo paradigma provocado por la crítica al neoliberalismo y el surgimiento de nuevos principios para el establecimiento de políticas de desarrollo. Al final del s. $X X$ se experimentaron una serie de interrupciones económicas en los países que habían adoptado la fórmula neoliberal dejando en evidencia que

no se estaban obteniendo los niveles de crecimiento esperados; son ejemplos de estas situaciones las crisis económicas y financieras en Rusia, Estados Unidos, América Latina y Asia, dejando claro que los mercados por sí solos no crean las condiciones de su proprio éxito.

Las conclusiones alcanzadas en esta etapa reconocen también que la actividad desregulada de los mercados ha sido ineficiente por lo que se requiere una intervención limitada de los Estados para corregir las interrupciones económicas consecuentes, así como los costos de las transacciones y las asimetrías en el manejo de la información. Los críticos de la segunda etapa argumentan que los proponentes de políticas internacionales de desarrollo no tuvieron en cuenta la capacidad de las instituciones locales, que fue un error establecer recetas de reforma legal trasplantadas de otras experiencias, que aún cuando promovían mejores prácticas, carecían del sentido de contexto local y que el enfoque exclusivo en el crecimiento económico que dominó igualmente las etapas del pensamiento sobre desarrollo, no condujo a la reducción de la pobreza.

Siguiendo a Trubek y Santos (Trubek y Santos, 2008), la escuela actual de Derecho y Desarrollo está re-elaborando sus líneas de pensamiento a partir de dos conclusiones principales: a) el reconocimiento de los límites del libre mercado y b)la ampliación del concepto de Desarrollo. A partir de esto, las proposiciones actuales están encaminadas a establecer regulaciones apropiadas a la actividad de mercado, sin menoscabar los beneficios de la libertad de comercio y el flujo de capitales, bienes y servicios, así como a re- conceptualizar el Desarrollo con 
el objeto de descentralizar el enfoque en el crecimiento económico para concentrarse en el "Desarrollo Humano".

Todas estas consideraciones están impactando el papel de la ley, la que ahora debe viabilizar la limitación apropiada de la actividad del mercado y asegurar el desarrollo humano, garantizar el enfoque de contexto local, la participación social y la reducción de la pobreza.

En los últimos tiempos la relación entre el derecho (expresado en la legislación) y el desarrollo se ha manifestado mediante una dinámica dialéctica de dos dimensiones ubicadas en extremos opuestos, por un lado la existencia de normas que legitiman y facilitan la realización de valores meramente económicos frente a la tendencia de crear normas que legitiman y facilitan la realización de otros valores (sociales y culturales, civiles y políticos).

Sobre la primera tendencia, a la que llamaremos "función economicista del Derecho en el Desarrollo", Douglass North (North, 2005) destaca la importancia de las instituciones económicas y políticas, la transformación de los derechos de propiedad y el efecto de la ideología en el análisis económico, con la finalidad de entender las determinantes del crecimiento económico.

Por su parte German Burgos (Burgos, 2009), interpretando a North establece que:

\footnotetext{
"las instituciones son ante todo reglas de juego entendidas como limitaciones humanamente concebidas que estructuran la interacción humana y cuya función principal es reducir la incertidumbre, o bajo otros términos ampliar la predictibilidad de la conducta humana y reducir los costos de transacción (North, 1993a). En otras palabras, las instituciones son reglas de acción destinadas a canalizar las conductas y a estabilizar las expectativas y reglas sociales (Pritzl, 2000)".
}

Sobre la segunda tendencia, a la que llamaremos "función integral del Derecho en el Desarrollo", se puede decir que es la que menos estudio posee, siendo imperativa la necesidad de suministrar mayores elementos teóricos que permitan un replanteamiento más operativo del rol del derecho en la consecución del Desarrollo Humano Sostenible.

David Trubek (Trubek, 2014) reconoce que la teoría del Derecho y Desarrollo debe lidiar con el creciente impacto de la globalización. Identifica tres fuerzas presentes: 1) la influencia de los modelos legales necesarios para la consecución de fondos internacionales; 2) la idea que los legisladores deben tener en cuenta el papel que juega la ley para determinar la competitividad de los países; en la medida que las estrategias de desarrollo dependan de inversión extranjera, en esa misma medida las leyes de esos países serán sometidas al escrutinio de los inversionistas quienes compararán los ambientes legales de las naciones antes de decidir la colocación de sus inversiones. Es así, que actualmente los inversionistas poseen muchas opciones de inversión y el conocimiento del estamento legal de un Estado es un factor determinante para la atracción de capital; 3) lo relacionado al crecimiento de las leyes transnacionales; de manera creciente el ordenamiento legal de un país es afectado por normas originadas fuera de sus fronteras.

Para reforzar lo anterior (Seidmann, Seidmann, \& Abeysekerer, 2001), establecen que desde el enfoque actual de la teoría del Desarrollo, la relación con el derecho cobra una relevancia considerable al referirse al marco de competencias para dirigir eficientemente los recursos económicos y sociales. Dicha capacidad en el manejo de los recursos depende en gran medida de la existencia de mecanismos de control para la toma de decisiones, las que no deben ser arbitrarias, sino reguladas por la ley y el Estado de Derecho, la rendición de cuentas, la transparencia y la participación ciudadana.

Los autores citados (Seidmann, Seidmann, \& Abeysekerer, 2001) reconocen que actualmente entre las categorías de Derecho y Desarrollo existe una relación complementaria en donde no se puede explicar el éxito de una sin el acompañamiento de la otra, teniendo como factor común de justificación e integración a la ley. Con ello se explica, por ejemplo, que los gobiernos no pueden proponer o cambiar patrones de uso de los recursos (sociales y económicos) sino por medio de la activación de un ambiente en donde los actores sociales relevantes deseen adherirse a otros mejores y más efectivos facilitados por la acción de esos mismos gobiernos desde donde se motiva el cambio hacia otros que incrementan la especialización y la productividad, mejorando el estado de bienestar social.

En este sentido se entiende que debe existir la permanente implementación de políticas diseñadas para facilitar el desarrollo. Si los gobiernos toman decisiones arbitrarias no fundadas en reglas, con suerte, tales decisiones producirán un ambiente para alcanzar el desarrollo. Por eso, las tendencias deterioradas de comunicación entre el derecho y el desarrollo causan dificultades para la formulación e implementación de sistemas legales que legitimen las acciones de los gobiernos en favor de mejorar las condiciones de sus habitantes.

Se ha identificado que en las sociedades contemporáneas la definición de políticas para el desarrollo debe justificarse en ley. Desde esta visión, la vinculación de la ley en la planificación del Desarrollo no es una coincidencia y tampoco es una cuestión de decisiones producto del sentido común. El Desarrollo implica cambio y en la medida en que las instituciones de los gobiernos prioricen sus acciones en función del desarrollo, en esa medida requieren el cambio de patrones de 
comportamiento que han resultado inefectivos por otros que permitan el uso inteligente y eficiente de los recursos de un Estado. Así, el ejercicio de poder para el logro de ese cambio se permite, en un Estado de Derecho, mediante la viabilidad que la ley le ofrece como fuente primaria de legitimación para el ejercicio de poder. La función de la ley en este contexto es resolver el problema de la inadecuación de patrones de comportamiento que han demostrado ser inefectivos en alcanzar el desarrollo.

\section{El Desarrollo Humano Sostenible como Derecho}

De acuerdo con Felipe Gómez Isa (Gómez Isa), el derecho al desarrollo constituye un derecho de muy reciente aparición, perteneciente a la tercera generación de Derechos Humanos también denominados derechos de la solidaridad. Éste derecho, junto con el derecho a la paz, al medio ambiente, entre otros, cierra por el momento el proceso de evolución de los derechos humanos iniciado en la revolución francesa.

Este mismo autor explica que la primera definición y caracterización del Derecho al Desarrollo, como derecho humano, se le debe al jurista senegalés Keba M'Baye (Uvin, 2004), quien en la sesión inaugural del Curso de Derechos Humanos de Estrassburgo en 1972, pronunció una conferencia sobre el derecho al desarrollo en el ámbito internacional. Posteriormente la Comisión de Derechos de la ONU reconoce por primera vez de manera oficial la existencia de un Derecho Humano al Desarrollo mediante la resolución 4(XXXIII), de 21 de febrero de 1977, en la que se le solicita al Secretario General de las Naciones Unidas que efectúe un estudio sobre "las dimensiones internacionales del Derecho al Desarrollo como Derecho Humano". El 2 de marzo de 1979, la Comisión de Derechos Humanos, en su resolución $5(X X X V)$ "reitera que el Derecho al Desarrollo es un Derecho Humano, y que la igualdad de oportunidades es una prerrogativa tanto de las naciones como de los individuos que forman las naciones".

En 1981, la Comisión de Derechos Humanos crea un Grupo de Trabajo de Expertos Gubernamentales para que elabore la caracterización del derecho al desarrollo como derecho humano, y sobre la redacción de un proyecto de Declaración sobre el Derecho al Desarrollo. La Asamblea General de la Organización de las Naciones Unidas (ONU) en 1986 aprobó la Declaración sobre el Derecho al Desarrollo, considerándolo como un derecho humano.

Esta decisión representó una nueva manera de abordar la realización de los ideales de las Naciones Unidas cuando proclama que: "La persona humana es el sujeto central del desarrollo y debe ser participante activo y el beneficiario del derecho al desarrollo" (ONU, 1986). Dicha Declaración reconoce, entre otras cosas, la interde- pendencia entre los derechos civiles y políticos con respecto a los derechos económicos, sociales y culturales y destaca la responsabilidad principal de los gobiernos en la realización del derecho al desarrollo.

Los trabajos posteriores que se siguieron a partir de la aprobación de la Declaración en 1986 establecieron la importancia de una estrategia a largo plazo que comprendiera el deber de los Estados de crear las condiciones internas conducentes a la realización del derecho al desarrollo, a través del establecimiento de políticas públicas y programas orientados a garantizar un equilibrio entre el crecimiento económico y el mejoramiento de las condiciones sociales, la participación de la población sin distinciones de ningún tipo, campañas de educación para que las personas tomaran mayor conciencia respecto a sus derechos y responsabilidades, la adopción de medidas más enérgicas a nivel internacional para crear las condiciones necesarias para la paz y seguridad.

Asimismo se establecieron como condiciones necesarias la reforma legislativa para garantizar que el derecho de los tratados tenga precedencia sobre el derecho interno, la ratificación de tratados de derechos humanos, la adopción de medidas económicas y sociales para evitar la exclusión de grupos marginalizados por causa de la pobreza extrema y la adopción de medidas para garantizar que los grupos pobres y vulnerables tengan acceso a los bienes productivos.

En la Conferencia de las Naciones Unidas sobre Medio Ambiente y Desarrollo celebrada en junio de 1992, conocida como "Declaración de Río", se vuelve a proclamar el Derecho al Desarrollo, vinculándolo de una forma muy estrecha con la protección del medio ambiente, es decir, el derecho al desarrollo se debe ejercer de tal forma que no ponga en peligro el ecosistema global. En ese sentido el principio número 3 de la Declaración (Departamento de Asuntos Económicos y Sociales, ONU, 1992) establece que "el Derecho al Desarrollo debe ejercerse en forma tal que responda equitativamente a las necesidades del desarrollo y ambientales de las generaciones presentes y futuras".

Siguiendo a (Gómez Isa) en la Conferencia Mundial de Derechos Humanos celebrada en Viena en junio de 1993 el debate sobre el derecho al desarrollo ocupó un lugar preeminente, en su párrafo subrayó que "la democracia, el desarrollo y el respeto de los Derechos Humanos y de las libertades fundamentales son conceptos interdependientes que se refuerzan mutuamente" y en el párrafo 10 se cita "la conferencia mundial de Derechos Humanos reafirma el Derecho al Desarrollo, según se proclama en la Declaración del Derecho al Desarrollo, como derecho universal, inalienable y como parte integrante de los derechos humanos fundamentales".

En 1997 la aprobación de Un Programa de Desarrollo por parte de la Asamblea General de la ONU afirmó la relación inter-vinculada del desarrollo y los derechos humanos. 


\section{Se reconoció que:}

"el respeto por todos los Derechos Humanos y libertades fundamentales, las instituciones democráticas y eficaces, la lucha contra la corrupción, la representación transparente y la gestión pública responsable, la participación popular, la independencia del sistema judicial, el imperio de la Ley y la paz civil están entre los fundamentos indispensables del Desarrollo"

(Escuela de Combia)

\section{Naturaleza Jurídica del Derecho al Desarrollo Humano Sostenible}

Miguel Ángel Contreras Nieto (Contreras Nieto, 2001) establece que el Derecho al Desarrollo presenta una particularidad dentro de la temática de derechos humanos, ya que ha mostrado una evolución inversa dentro de este campo, esto es, el reconocimiento y tutela de los derechos Hhmanos de primera y segunda generación, ha tenido origen en ordenamientos de algunos Estados, para de allí proyectarse al plano mundial, recogidos por la comunidad internacional representada en la Organización de las Naciones Unidas y por casi todos los países que los han incorporado a su normativa particular. $A$ diferencia de ello, el reconocimiento y protección del derecho al desarrollo se gestó originalmente a nivel internacional para pasar con posterioridad al ámbito interno de los países.

El autor citado, a su vez, hace una interesante recopilación de diferentes conceptos sobre el Derecho al Desarrollo, entre los cuales se destacan:

1. En 1972, Keba M'Baye, también citado en (Gómez Isa) y (Uvin, 2004) sostuvo que "el desarrollo es el derecho de todo hombre. Cada hombre tiene derecho de vivir y el derecho de vivir mejor".

2. En 1972, Juan Antonio Carillo, citado a su vez del Documento denominado "Estudios sobre Derechos Humanos" de la Editorial Jurídica Venezolana, estableció que:

"el Derecho al Desarrollo es un Derecho Humano y un derecho de los pueblos, lo que trae consigo el corolario de que todos los hombres y todos los pueblos, sin distinción, han de contribuir a una empresa común de la humanidad. Entendido como crecimiento más cambio, el desarrollo y el derecho al desarrollo como derecho humano constituyen un factor revolucionario en la vieja estructura del Derecho Internacional público, que en su proceso de socialización y democratización, no hace otra cosa que liberarse y humanizarse".

3. En 1975, Héctor Gros Espiell, citado a su vez del Documento denominado "Estudios sobre Derechos Humanos" de la Editorial Jurídica Venezolana, expresó: "el derecho al desarrollo como Derecho de los Estados y de los pueblos debe ineludiblemente fundarse en el reconocimiento del derecho de todo hombre a una vida libre y digna dentro de la comunidad. Todo ser humano tiene el derecho a vivir, lo implica el derecho a aspirar a una existencia cada vez mejor. Este derecho al pleno desarrollo individual -que ha permitido que con razón se califique al derecho al desarrollo como un derecho humano fundamental- sirve de base, al mismo tiempo que condiciona e implica el derecho de los pueblos y de los Estados en vías de desarrollo al desarrollo. El progreso de estos sólo se justifica en cuanto el desarrollo sirva para mejorar la condición económica, social y cultural de cada persona humana".

4. En la década de los años ochenta, se reconoció el Derecho al Desarrollo como derecho de la persona humana, sumándose a la noción colectiva, la cual fue positivizada convencionalmente por primera ocasión en la Carta Africana de los Derechos Humanos y de los Pueblos de 1981, instrumento en el que se estableció que todos los pueblos tienen el derecho al desarrollo económico, social y cultural, que coincida con su libertad e identidad.

5. De acuerdo al punto de vista de Miguel Concha Malo, citado a su vez de "Los derechos humanos como precondición de la democracia, el desarrollo y la paz" de la Revista Justicia y Paz, edición abril/junio, 1994, México, el desarrollo es:

"el derecho al despliegue de todas las facultades del ser humano y al disfrute de una vida que sea completamente satisfactoria para el individuo y para su sociedad; al igual que los demás derechos humanos, exige como requisito indispensable para su vigencia el reconocimiento y vigencia de todos los demás derechos humanos. Implica la existencia de un régimen democrático que estimule la participación ciudadana en los asuntos públicos, en lo político; de un sistema económico que brinde satisfacción a las crecientes necesidades de toda la población; de un sistema de impartición de justicia que sea imparcial, probo, ágil y eficaz; etc. Y esta relación es dialéctica, si tomamos en cuenta que una sociedad compuesta por individuos crecientemente satisfechos en todos los aspectos de la vida, es una sociedad que al mismo tiempo que exige mejores condiciones, aporta lo mejor de sí para ese fin. En otras palabras, que el motor del desarrollo humano debe ser el propio desarrollo humano".

Siguiendo a (Contreras Nieto, 2001) jurídicamente el Derecho al Desarrollo se configura por tres elementos: el primero, un sujeto activo o titular, que es todo ser humano, considerado individual o colectivamente, a quien se le atribuye un derecho; el segundo, un sujeto pasivo, frente a quien se hace exigible el goce y ejercicio de este derecho, el cual tiene la obligación, bien sea positiva, de dar o hacer algo, o negativa, de no hacer, para la satisfacción al derecho del sujeto activo, normalmente identificado en el Estado, la comunidad internacional o los países desarrollados; y el tercero, un objeto consistente en el desarrollo integral del sujeto activo de este derecho. 


\section{Sobre el Enfoque de Derechos Humanos}

Jacob Kirkemann y Tomas Martin (Kirkemann \& Martin, 2007), definen el Enfoque de Derechos Humanos (EDH) como un marco de referencia que integra las normas, los principios, estándares y metas de los derechos humanos reconocidos internacionalmente para ser aplicados en la planificación del desarrollo.

Esta postura reconoce la pobreza como una situación de injusticia, que implica la marginalización, la discriminación y la explotación como sus principales causas; en este contexto, la pobreza no es considerada como una responsabilidad individual, sino que se explica a partir del reconocimiento de relaciones entre sujetos de derecho y sujetos de obligaciones. La dinámica central del EDH es identificar las causas de la pobreza, empoderando a los sujetos de derecho a exigir su cumplimiento y a activar a los sujetos de obligaciones a su cumplimiento.

Las consideraciones en las que se apoya el EDH llama la atención de un buen número de condiciones relacionadas con la pobreza y el desarrollo, entre ellas:

1. La consideración del Estado y la gobernabilidad como elementos centrales del desarrollo, basada en la inter-relación con sus ciudadanos en términos de derechos y obligaciones. Bajo esta idea, el EDH reconoce la obligación básica de los Estados de cuidar de las personas más vulnerables, incluyendo aquellas que no pueden demandar sus derechos por sí solas.

2. El reconocimiento que la pobreza es una violación de los derechos humanos y que ésta a la vez es la causa de las trasgresión de aquellos. Así, la consideración de la pobreza desde los lentes de la justicia se constituye en una realidad impuesta sobre las personas como una acción activa (y en ciertos momentos pasiva por omisión) de discriminación y marginalización; y,

3. El reconocimiento cada vez más claro que la pobreza no consiste únicamente en la negación de las necesidades económicas sino que transgrede cuestiones más fundamentales y complejas de la dignidad de las personas. Esta condición implica también que la pobreza no es un hecho atribuible a circunstancias o capacidades individuales, sino que debe ser percibida como derivación del ejercicioinequitativo de poder implícito en los contextos locales, nacionales y globales.

En el mismo sentido Thomas Pogge (Pogge, 2005) establece que para incorporar el concepto de justicia en las decisiones de las instituciones sociales que procuran el desarrollo, deben considerarse las siguientes cuestiones:
1. El criterio universal de Derechos Humanos como elemento fundamentador de las instituciones que procuran el desarrollo debe incorporarse en el lenguaje de los "medios" para el desarrollo, en lugar del lenguaje de los "componentes" del desarrollo. De acuerdo a esta idea, la alimentación, la vivienda, el trabajo, el vestido, las libertades básicas, entre otros, constituyen medios importantes para el desarrollo que las instituciones deben asegurar a todos. Esta propuesta facilitaría la transición de los desacuerdos colectivos sobre el concepto de desarrollo y representaría cierto respeto por las diversas expresiones de culturas, al mediar por instituciones sociales que podrían aceptar personas de diferentes procedencias, historias y tradiciones, quienes representan a su vez una variedad de concepciones de Desarrollo más específicas.

2. Asimismo, el criterio universal de Derechos Humanos, debe ser contextual. En vez de definirse como el punto más alto a alcanzar en una escala no definida, se debería definir como una meta razonable, compatible con una variedad internacional de esquemas institucionales a los que se les demanda tratar a las personas vinculadas por ellos de una forma digna.

3. No se deben interpretar los requisitos del criterio universal de los Derechos Humanos como criterios exhaustivos. Dichos requisitos deben dejar la oportunidad de que las sociedades establezcan sus propios criterios más exigentes sobre sus propias instituciones nacionales, e incluso la posibilidad de que cuestionen las instituciones globales o extranjeras a la luz de criterios más retadores; $y$,

4. Las consideraciones adicionales que introduzcan estos criterios más retadores no deben poner en riesgo la universalidad de los criterios básicos de contexto, no se debe permitir sobre estos en caso de conflicto. Es así que los criterios universales deberían ocupar un lugar preferencial frente a cualquier criterio nacional más ambicioso.

Kirkemann y Martin (Kirkemann \& Martin, 2007), también establecen que la aplicación del EDH contribuye a mejorar la situación de las personas ya que tiene como punto de referencia sus necesidades, problemas y potencialidades; en ese sentido coincide con todas las circunstancias consideradas en los iniciativas de desarrollo tales como la alimentación, acceso al agua, vivienda, salud, educación, seguridad y en general, la libertad para el desarrollo humano; no obstante, para desde esta visión se establece el principio que las personas tienen derechos inalienables a las circunstancias anteriormente mencionadas y que la privación de las mismas es una negación a ese reconocimiento de derechos. En otras palabras, el acceso al agua potable (por ejemplo) no se considera únicamente como una necesidad humana sino como un objeto de derecho en relación a la condición de ser persona (sujeto de derecho). 
Asimismo, es necesario destacar que aún cuando las "necesidades" humanas básicas son la base de los derechos humanos hay notables diferencias en considerarlas precisamente "necesidades" o "derechos", por lo tanto hay que establecer que:

1. Los derechos humanos van más allá de la noción de las necesidades físicas e incluyen una perspectiva holística de las personas en términos de sus capacidades civiles, políticas, económicas, sociales y culturales.

2. El EDH se fundamenta en la relación derecho-obligación; los derechos no pueden establecerse sin considerar el nexo de quien tiene la obligación de realizarlos; $y$,

3. Bajo el enfoque de necesidades se espera que las personas beneficiarias sean "agradecidas" por el otorgamiento o realización de sus expectativas, sin embargo, bajo el EDH se trata de la realización de las expectativas a las que las personas tienen derecho. Bajo esta idea, es importante tener en cuenta que la promoción del desarrollo no se hace a favor de los "necesitados" sino a favor de las personas marginalizadas con iguales aptitudes para reclamar la realización de oportunidades que reduzcan su pobreza y la injusticia a las que han estado expuestas.

\section{La Complejidad de la Pobreza desde el Enfoque de Derechos Humanos}

Normalmente, las iniciativas de desarrollo se impulsan a partir de la proyección de mejores condiciones de vida, de esa manera se entiende que la acción lógica de los agentes de desarrollo frente a las personas que tienen hambre es darles alimento, pero si a la vez se descubre que las deficiencias en el acceso a los alimentos se debe a las limitaciones de sus pequeñas tierras de cultivo, la acción es darles más tierra para cultivar. Si a esto se agrega información adicional en el sentido que el acceso a la tierra para cultivo es limitado por que las autoridades locales decidieron un destino diferente para esas parcelas o si se identifica que el propietario de la tierra es una mujer, que por su condición de sexo no pudo ser beneficiaria por los esquemas de redistribución de las autoridades, se comprenderá que el problema se agudiza en la medida en que se profundiza en la identificación de las causas que han impedido el acceso adecuado a aquellos alimentos. Las soluciones simples, sólo tocan la superficie de las realidades que provocan la pobreza.

Una vez que se acepta que la pobreza es un fenómeno complejo, se comprende la necesidad de atender esta condición mediante soluciones que puedan comprehender esa complejidad. Al respecto, el EDH ofrece perspectivas capaces de embargar todas sus causas civiles, políticas, económicas, sociales y culturales de la pobreza; bajo dicho enfoque, no sólo es entendida como la deficiencia en el acceso a recursos que puede ser solucionada mediante la provisión de cosas materiales, ya que aún cuando los recursos están disponibles, su acceso puede ser limitado a los pobres en razón de quiénes son, dónde viven, o simplemente por actos de omisión o negligencia. Esta forma de discriminación puede ser el resultado de normas, valores sociales y políticas inequitativas vigentes, causantes de estigmatización y marginalización, lo que lleva a concluir que la pobreza es una acción ejecutada contra las personas.

\section{Los Sujetos de Derechos y los Sujetos de Obligaciones}

Para entender la relación entre "sujetos de derechos" y "sujetos de obligaciones" en el EDH, referida anteriormente, es necesario tener en cuenta algunos aspectos conceptuales de los derechos humanos, a saber: que pueden ser descritos como las garantías legales universales para la protección de las personas y grupos frente a las acciones y omisiones que puedan afectar su libertad y su dignidad, constituyen los estándares mínimos básicos para la realización de las necesidades humanas, de carácter universal e inalienables, intrínsecos a la condición de personas, intemporales e irrenunciables, indivisibles e interdependientes.

Los derechos humanos internacionalmente reconocidos nacen de los convenios internacionales y las declaraciones que los definen y, que a su vez, establecen los mecanismos de protección a los cuales los Estados se adhieren bajo la tesis fundamental que la legitimidad de dichos Estados está cimentada en el respeto, protección y realización que hagan de esos derechos a favor de todas las personas.

Una de las premisas fundamentales para la comprensión de los derechos humanos es el reconocimiento de que todas las personas "tienen derecho" al ejercicio de aquellos, y consecuentemente, se ponen en una situación relacional frente a (sujetos de obligaciones) quienes tienen la obligación de garantizarlos. Todo sujeto de derecho tiene derecho al ejercicio de sus derechos, así como a demandar su cumplimiento cuando son restringidos y a exigir la transparencia y la responsabilidad frente a quienes ostentan el deber de realizarlos.

De acuerdo a la teoría de los derechos humanos internacionalmente reconocida, la responsabilidad de su realización descansa en el Estado, los Parlamentos, las instituciones de gobierno, autoridades locales, tribunales de justicia, autoridades de seguridad y todos aquellos que representen funciones públicas debidamente delegadas. 


\section{Conclusiones}

La vida jurídica del país se regula por un número cercano a las 2000 leyes. Para establecer un contraste sobre la importancia de ese número se debe mencionar, por ejemplo, que en la Facultad de Derecho de la UNAH, los estudiantes normalmente tienen acceso y analizan un número muy inferior al total de las leyes identificadas; para el caso, en el ámbito del Derecho Civil (considerado como la columna vertebral del Derecho) se identificaron 317 leyes de los cuales las más consultadas en el transcurso de la carrera son el Código Civil, Código Procesal Civil, Código del Notariado y la Ley de Propiedad, es decir el $1 \%$ del total en esa categoría y el $0.2 \%$ aproximadamente del total general. Esta inferencia puede extenderse al campo del Derecho Mercantil y el Administrativo-Fiscal que constituyen los ámbitos con mayor número de cuerpos normativos.

En futuros procesos de investigación corresponderá indagar sobre las leyes que más se invocan en la actividad judicial y en el ámbito de la justicia administrativa.

La mayor cantidad de leyes en Honduras están concentradas en los ámbitos administrativo- fiscal, mercantil y civil; y en cuarto lugar lo penal. A partir de este dato pueden establecerse una serie de inferencias importantes para la reflexión propuesta en este trabajo, a saber, en primer lugar, es necesario considerar que estos cuerpos normativos regulan en esencia la dimensión económica de la sociedad; para el caso, desde el punto de vista fiscal, se establecen los criterios que orientan al Estado en su función de recaudación de impuestos, la que a su vez está relacionada con la capacidad adquisitiva de los contribuyentes. La imposición de tributos es una prerrogativa de los Estados basada en la contribución que todos los ciudadanos de un país están llamados a prestar para mejorar las condiciones de vida social de la comunidad a la que pertenecen.

En el caso de las esferas mercantil y civil, sus contenidos normativos regulan las actividades de las personas en su ámbito privado, y en especial, en la relación con el ejercicio del derecho de propiedad. Esto quiere decir que históricamente, la preocupación del legislador ha estado concentrada en la actividad económica de las personas que regula mediante las normas que promulga, ya sea en razón del ejercicio del derecho de propiedad para el uso, goce y disposición privada de los bienes sobre los que ejerce este Derecho, o para la regulación de la explotación masiva del mismo y/o para la imposición de tributos en razón del patrimonio de las personas.

En segundo lugar, en contraste con lo expresado en el párrafo anterior, las leyes con contenido social, de promoción de igualdad de oportunidades, de atención a los pobres, son las de menor presencia en el universo legal hondureño. Los primeros cuerpos normativos con contenidos sociales explícitos se generaron después del consenso internacional reconocido en la Declaración Universal de los Derechos Humanos, tal es el caso del Código Laboral (1957) y las Constituciones de 1957,1965 y $1982^{5}$.

Por otro lado, de acuerdo al análisis comparativo del proceso de creación de leyes establecido en las catorce Constituciones que han regido la vida republicana de Honduras para dar cuenta de la evolución histórica de esta atribución reconocida al Poder Legislativo, llama la atención que en ninguno de los preámbulos ni en la parte dispositiva de las Constituciones estudiadas se encuentra incorporada la palabra "Desarrollo" como expresión de una aspiración o un objetivo de la organización social derivada del acto constitutivo.

A la vez, puede decirse que el hallazgo más importante derivado de este análisis comparativo es la socialización del proceso de creación de normas, se ha pasado de un modelo en el que la atribución de presentar iniciativas de ley era exclusiva de los miembros del Congreso Nacional a otro (actual y vigente) en el que se concede, bajo ciertas condiciones, que los ciudadanos organizados promuevan iniciativas de creación o reforma de normas legales.

Si la dinámica social es compleja, la organización jurídica de la sociedad será igualmente compleja. El derecho como fenómeno social que se expresa en normas, hechos y/o valores se deriva de la aspiración social de consolidar la paz. La convivencia armónica que el derecho desea alcanzar no es un resultado espontáneo derivado de la condición biológica del ser humano, si no de su esencia social, por tanto voluntaria. El derecho nace de la voluntad de todos los individuos que conforman una comunidad y que buscan conscientemente establecer un contrato social que les garantice las condiciones y oportunidades de su despliegue personal y colectivo.

Las normas legales representan una sola dimensión del derecho y en su contenido reflejan la impronta del acto social que las constituye. No existirá derecho ni normas legales para el último hombre que habite el planeta, mientras exista una comunidad de dos o más personas, ahí existirá derecho y por ende normas jurídicas, creadas mediante consensos recíprocos y complejos porque buscarán garantizar las aspiraciones de desarrollo de los individuos que desean organizar su vida social conforme a ellas.

La creación de las normas legales, entre otras muchas cosas, será siempre compleja, como lo es el acto social que las origina o que las demanda. Para elevar la calidad técnica de la

5-El Código Civil tiene 110 años de vigencia, fue promulgado en 1906 y es de las normas que menos reformas ha sufrido, salvo lo relativo a la regulación de los temas del Derecho de Familia que fueron reformados con la promulgación del Código de Familia en 1984, es decir 78 años después de su entrada en vigencia. 


\section{Legislación con enfoque de Derechos Humanos para la gestión de Desarrollo Humano Sostenible en Honduras}

norma legal que pretende regular un determinado fenómeno social es necesario reconocerle "mutabilidad originaria" que le permita adaptarse a los hechos y circunstancias en una dimensión histórica, de constante transformación.

Ante tal reconocimiento no se puede seguir forzando la función organizadora del derecho exclusivamente en su dimensión normativa, es necesario distinguir que la norma legal tiene sentido cuando responde a un hecho social que la origina o la deriva y cuando tiende a la realización de un valor que igualmente la origina o la deriva.

Actualmente, la dimensión axiológica del derecho está fundamentada en el reconocimiento internacional de los derechos humanos. La comunidad internacional progresivamente ha ido asumiendo este consenso como la base de referencia mínima para la consolidación de la paz y en ese aprendizaje ha distinguido derechos universales en razón de la esencia individual y social de los seres humanos, y en esa misma línea ha reconocido que el desarrollo humano corresponde a una categoría jurídica de norma, hecho y valor. Es decir, todo derecho humano y particularmente el derecho humano al desarrollo es una relación jurídica (de derechos y obligaciones), es un fenómeno social porque implica el pleno despliegue de las potencialidades humanas y sociales y es un valor porque orienta el esfuerzo social de organización para la consecución de la paz.

Por tanto, toda labor jurídica que implique el establecimiento de normas legales para la organización social debe propender a la consolidación inexcusable de los derechos humanos, lo que a su vez conlleva a la consolidación del derecho humano al Desarrollo Humano Sostenible.

\section{Bibliografía}

1. Anand, S., \& Sen, A. (Marzo de 1994). United Nation Development Programme. Recuperado el 14 de Febrero de 2016, de Human Development Reports: http://hdr. undp.org/es/content/sustainable-human- developmentconcepts-and-priorities

2. Burgos, G. (2009). Estado de Derecho y Globalización: El Banco Mundial y las reformas institucionales en América Latina. Bogotá, Colombia: ILSA-UNIJUS.

3. Cepeda, B. P. (1999). Metodología Jurídica . México: Oxford University Press.

4. Contreras Nieto, M. (2001). El Derecho al Desarrollo como Derecho Humano. Toluca, México: Instituto Literario. Comisión de Derechos Humanos del Estado de México.

5. Donnelly, J. (2003). Universal Human Rights in theory and practice. Nueva York, EE.UU: Cornell University Press.
6. Gómez Isa, F. (s.f.). Observatorio de Derechos Económicos, Sociales y Culturales. Recuperado el 9 de Diciembre de 2015, de http://observatoridesc.org/files/ cap11.pdf

7. Grosso y Svetaz, B. M. (2001). Técnica Legislativa en Centroamérica y República Dominicana. San José, Costa Rica: Instituto Interamericano de Derechos Humanos.

8. Kirkemann, \& Martin, J. y. (2007). Applying a rights based approach. An inspirational guide for civil society. Copenhague, Dinamarca: The Danish Institute for Human Rights.

9. Maynez, E. G. (1961). Introducción al Estudio del Derecho. México: Porrúa.

10. North, D. (2005). Understanding the process of economic change. Woodstock, Oxfordshire: Princeton University Press.

11. Novoa Monreal, E. (1975). Repercusiones de las nuevas funciones del Estado en el Derecho. En E. Novoa Monreal, El Derecho como obstáculo al cambio social (pág. 123). México: Siglo XXI Editores.

12. Peces-Barba, G. (1980). Derechos Fundamentales. Madrid: Latina Universitaria.

13. Pogge, T. (2005). La Pobreza en el Mundo y los Derechos Humanos. Barcelona, España: Paidós.

14. Reale, M. (1989). Introducción al estudio del Derecho. Madrid: Pirámide.

15. Rodríguez Braun, C. (2005). Estudio Preliminar. En A. Smith, La riqueza de las naciones (pág. 15). Madrid, España: Alianza Editorial.

16. Secretaría de Gobernación y Justicia. (2005).Las Constituciones de Honduras. Tegucigalpa,Honduras: ENAG.

17. Seidmann, Seidmann, \& Abeysekerer, R. A. (2001). Legislative Drafting for Democratic Social Change. A Manual for Drafters. Boston, USA: Boston University Press.

18. Serrano, A. (2007). Primer Módulo. Programa Doctoral en Ciencias Soicales con orientación en gestión del Desarrollo. Tegucigalpa: UNAH.

19. Sistema de Naciones Unidas-Honduras. (2008). Guía metodológica para el enfoque de Derechos Humanos en políticas públicas y planes sectoriales. tegucigalpa, Honduras: Casa de las Naciones Unidas.

20. Trubek y Santos, D. y. (2008). The New Law and Economic Development. A Critical Appraisal. Nueva York: Cambridge University Press. 
21. Trubek, D. (2014). Law and Development: 40 years after Scholars ins Self Estrangement. A Preliminary Reviem. Legal Scholarship Network , 2-16.

22. Uvin, P. (2004). Human Rights and Development. Bloomfield, CT: Kumarian Press, Inc.

23. Witker y Larios, J. R. (2002). Metodología Jurídica. México: McGraw Hill.

\section{Referencias institucionales:}

1. Asamblea Nacional Constituyente. (1981). Decreto Número 131. Constitución de la República. Honduras.

2. Congreso Nacional. (2013). Decreto 190-2012.Ley de mecanismos de participacion ciudadana. Honduras.

3. Congreso Nacional. (2013). Decreto 363-2013.Ley Orgánica del Poder Legislativo . Honduras.

4. Congreso Nacional. (1906). Decreto Número 76. Código Civil . Honduras.

5. Departamento de Asuntos Económicos y Sociales, ONU. (1992). Recuperado el Octubre de 2015,de División de Desarrollo Sostenible: http://www.un.org/spanish/esa/ sustdev/agenda21/riodeclaration.htm

6. Escuela Colombia. (s.f.). Biblioteca Plan Colombia. Recuperado el 8 de Octubre de 2015, de http://ilsa.org.co:81/ biblioteca/dwnlds/utiles/deuda/Deud a/ati/b_Deuda_Nacional/DERECHODESARROLLO.htm

7. ONU. (1948). Declaración Universal de los Derechos Humanos. Nueva York, EUA: ONU.

8. ONU, A. G. (4 de Diciembre de 1986). Declaración sobre el Derecho al Desarrollo.

9. Programa de las Naciones Unidas para el Desarrollo, PNUD). (1990). United Nations Development Programme. Recuperado el Febrero de 2016, de Human Development Reports: http://hdr.undp.org/es/content/informe-sobre-desarrollo- humano-1990

10. Secretaría de Gobernación y Justicia. (2005).Las Constituciones de Honduras. Tegucigalpa, Honduras: ENAG.

11. Sistema de Naciones Unidas-Honduras. (2008). Guía metodológica para el enfoque de Derechos Humanos en políticas públicas y planes sectoriales. tegucigalpa, Honduras: Casa de las Naciones Unidas.

12. Wikipedia. (8 de Noviembre de 2014). Pactos de Nueva York. Recuperado el 14 de Octubre de 2015, de https :/l es. wikipedia.org/wiki/Pactos_de_Nueva_Y ork

Convenios internacionales consultados
1. Declaración Universal de Derechos Humanos. Adoptada por la Asamblea General de la Organización de Naciones Unidas en su resolución 217 A (III) de 10 de diciembre de 1948.

2. Pacto Internacional de los Derechos Económicos, Sociales y Culturales. Acuerdo No. 10 del 22 de Abril de 1980. Publicado en el Diario Oficial "La Gaceta" el 30 de julio de 1980.

3. Pacto Internacional de Derechos Civiles y Políticos. Decreto No. 64-95. Publicado en el Diario Oficial "La Gaceta" el 24 de junio de 1997.

4. Convención Internacional sobre la eliminación de todas las formas de discriminación racial. Decreto No. 61-2002. Publicado en el Diario Oficial "La Gaceta" el 6 de julio de 2002

5. Convención sobre la eliminación de todas las formas de discriminación contra la mujer. Decreto. No. 979. Publicado en el Diario Oficial "La Gaceta" el 10 de septiembre de 1980 .

6. Convención contra la tortura y otros tratos crueles, inhumanos o degradantes. Decreto no. 47-96. Publicado en el Diario Oficial "La Gaceta" el 19 de octubre de 1996.

7. Convención sobre los Derechos del Niño. Decreto No. 75-91. Publicado en el Diario Oficial "La Gaceta" el 10 de agosto de 1990.

8. Convención Americana sobre Derechos Humanos. Decreto No. 523. Publicado en el Diario Oficial "La Gaceta" el 1 de septiembre de 1977.

9. Protocolo Adicional a la Convención Americana sobre Derechos relativos a los Derechos Económicos, Sociales y Culturales. No ratificada.

10. Convención interamericana para prevenir, sancionar y erradicar la violencia contra la mujer. Decreto 72-95. Publicado en el Diario Oficial "La Gaceta" el 14 de junio de 1995

11. Convenio 87-OIT relativo a la libertad sindical y a la protección del derecho de sindicación. Decreto No. 17. Publicado en el Diario Oficial "La Gaceta" el 24 de mayo de 1956.

12. Convenio 98-OIT relativo a la aplicación de los principios de sindicación y negociación colectiva. Decreto No. 18. Publicado en el Diario Oficial "La Gaceta" el 24 de mayo de 1958.

13. Convenio 105-OIT relativo a la abolición del trabajo forzoso. Decreto No. 39. Publicado en el Diario Oficial "La Gaceta" el 23 de mayo de 1958. 
14. Convenio 138-OIT sobre la edad mínima de admisión al empleo. Decreto No. 952. Publicado en el Diario Oficial "La Gaceta" el 8 de julio de 1980.

15. Convenio 169-OIT sobre los pueblos indígenas y tribales en países independientes. Decreto No. 26-94. Publicado en el Diario Oficial "La Gaceta" el 30 de julio de 1994.

16. Convenio 182-OIT sobre las peores formas de trabajo infantil y la acción inmediata para su eliminación. Decreto No. 62-2001. Publicado en el Diario Oficial "La Gaceta" el 28 de julio de 2001. 